\title{
An Adaptive MAC Layer Protocol for ATM-based LEO Satellite Networks (Extended Abstract)
}

\author{
Marc Emmelmann* \\ ${ }^{*}$ Corresponding Author \\ Fraunhofer Institut FOKUS \\ Competence Center for Advanced Network Technologies \\ Berlin, Germany \\ Email: emmelmann@ieee.org \\ Hermann Bischl \\ German Aerospace Center (DLR) \\ Institute of Communications and Navigation \\ Oberpfaffenhofen, Germany \\ Email: Hermann.Bischl@dlr.de \\ (Invited Paper) \\ Abstract
}

German Aerospace (DLR), Fraunhofer FOKUS.cats, and Tesat-Spacecom have designed a future multimedia ATM-based LEO satellite network. Part of the development are an adaptive MAC and FEC scheme which is presented in this paper. The FEC implementation switches on the fly during a connection between several FEC and modulation modes to guarantee a maximum ATM cell error rate of $10^{-6}$. In order to prevent influences of the changing FEC scheme on the user-level data rate, the MAC dynamically changes its packet lengths. Additionally, to minimize contention on the up-link, the MAC structures its TDMA frame into a fixed assigned part and a contention specific part. The boarder in between the two is dynamically altered according to the current link utilization.

EDAS Paper ID: 112966185

to be published at:

Vehicular Technology Conference - Broadband Satellite Networks Track 



\section{An Adaptive MAC Layer Protocol for ATM-based LEO Satellite Networks (Extended Abstract)}

\section{INTRODUCTION}

Future satellite networks for multimedia communication will be seamlessly integrated into terrestrial networks. Therefore, they have to support inherently any kinds of quality of service. The design of such a - most possibly ATM-, all-IP, or MPLS-based - broadband multimedia satellite system is a technically challenging task esp. for non geostationary (NGSO) constellations. Even though most of the presently proposed broadband satellite systems are GEO utilizing DVB$\mathrm{S}$ and DVB-RCS, future satellite networks will incorporate NGSO constellations; regenerative payloads; and on-board switching, routing, and processing. The satellite community's view of seeing a "network in the sky" to consist of a bent pipe, transparent bit-stream channel continuously moves towards this new kind of network architecture. [1] As a result, new MAC and FEC schemes have to be considered.

This paper discusses a MAC protocol and FEC scheme which were developed by German Aerospace Center (DLR) and Fraunhofer FOKUS.cats for such a future broadband satellite network. [2] The MAC protocol has been designed for a better utilization of the available bandwidth and supports different QoS classes. The variable burst length of the MAC scheme allows not only a verly flexible allocation of the radio resources to the terminals, but also adaptive error control coding and moduation. A performance analysis shows that adaptive coding and modulation offers high availability even in Ka-band systems suffering from high rain attenuation.

The paper is structured as follows: Section II deals with the technical aspects of the designed MAC protocol including scheduling and FEC schemes. Section III afterwards explains how the different protocol entities are separated for implementation purposes for the demonstrator. Some measurements to verify the protocol functionality are outlined.

\section{Protocol Design}

The most natural approach to develop a satellite system supporting ATM is to develop a MAC layer which inherently supports different QoS classes. Several constrains deriving from a NGSO satellite constellation and the ATM protocol itself influenced the design, i.e:

- the lack of a dedicated QoS field within an ATM-cell,

- variable propagation delays and swiftly changing channel error rates due to changes in the satellite's elevation angle, and

- the severe impact of rain attenuation and shadowing due to vehicular's movements.

The first is solved by an adaptive MAC framing structure and an introduced layer management entity while the latter is handled by an adaptive forward error correction scheme.
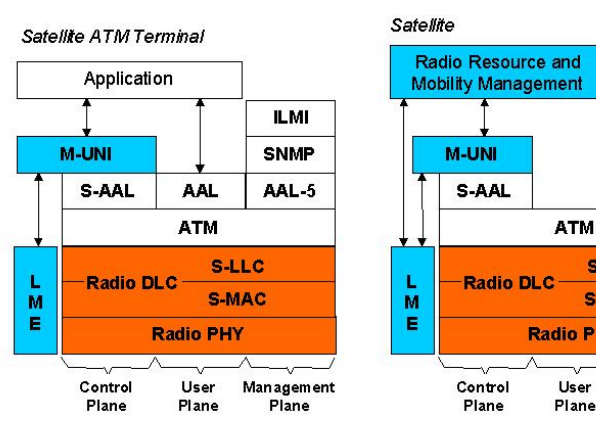

Fig. 1. ATM-Sat Protocol Stack

\section{A. Medium Access Control and Scheduling}

1) Protocol Stack: In terrestrial ATM networks, service parameters are announced during the connection set-up phase together with a unique VPI/VCI value. The only way to identify a connection's QoS parameters afterwards is through its VPI/VCI value. Therefore the MAC layer has to embed any kind of lookup table to guarantee $\mathrm{QoS}$ constrains for the different connections. Figure 1 depicts the protocol stack in which the layer management entity (LME) connects UNI and MAC and bypasses service parameters during connection establishment.

2) MAC Framing Structure: The up- and down-link are duplexed by FDD which is most convenient in power limited systems. Up- and down-link themselves are structured into fixed length frames. The frame length is $24 \mathrm{~ms}$ and is a direct result to easily support a typical voice specific data rate of $16 \mathrm{kbit} / \mathrm{s}$.

The primary goal of the MAC for the up-link direction is to reduce or even eliminate contention. This is achieved by dividing the up-link TDMA frame into two areas: a reservation area to transmit data of existing connections in previously assigned slots, and a contention area which is used for initial connection setup or bandwidth requests in case a terminal has no more resources in the reservation area. The boundary between the two areas adapts to the current link utilization and therefore reduces the contention probability within periods of low transmission activity. The reservation 


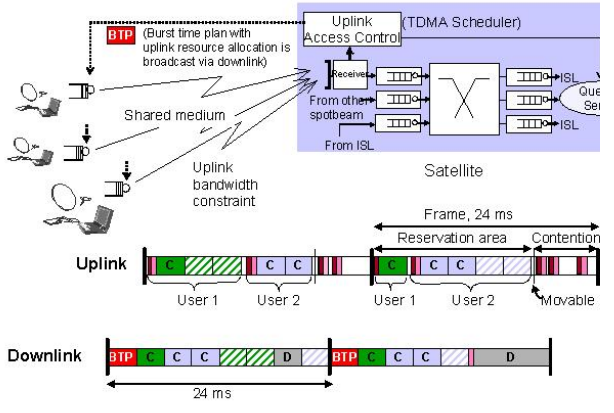

WIA UBR cells C C CBR cells 【MAC signalling (eg, resource request) IPreamble [

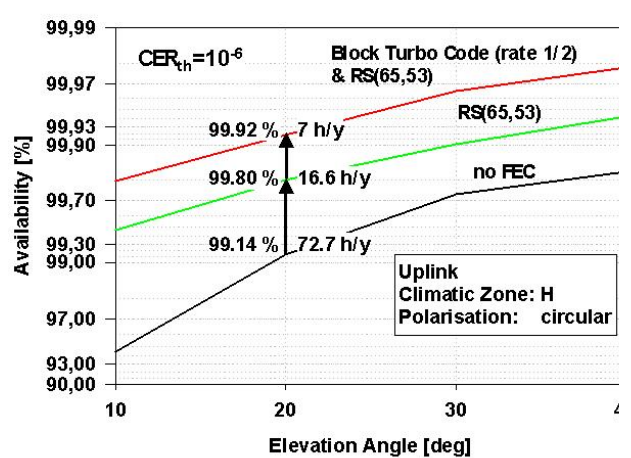

Fig. 3. TDMA Scheduler \& MAC Up- and Down-link Frame Structure
Fig. 2. TDMA Scheduler \& MAC Up- and Down-link Frame Structure

area is divided into slots which are assigned to each user on a frame-by-frame basis as illustrated in Fig. 2. The assignment is constrained by the fact that each user transmits all its granted slots in a burst thus reducing the number of required preamble sequences and guard times to one for each user. The delay variance introduced by this MAC scheme is flattened by a traffic shaper located in the receiving MAC to support constant bit rate (CBR) connections. The hereby introduced additional (const.) delay of one TDMA frame is well acceptable even for real-time applications.

The down-link frame is simply divided into slots containing either unspecified bit rate (UBR) or constant bit rate (CBR) cells. Additionally, it always starts with a burst transmission plan (BTP) which holds information on the structure of the next up-link TDMA frame.

3) Up-link Scheduler: The up-link scheduler is located on board the satellite. It assigns fixed resource allocations to all CBR connections with highest priority. Remaining resources are assigned to UBR with a minimum cell rate (MCR) connections based on a weighted round robin algorithm (weights set to the MCR). Left-over resources are equally divided between all remaining UBR and UBR with MCR connections.

\section{B. Forward Error Correction Schemes}

The designed satellite system utilizes the Ka-band which mainly suffers from high rain attenuation and - in case of moving vehiculars - also from high signal shadowing due to obstacles like trees or buildings. As for this project vehicles are equipped with directional antennas, multipath fading is slight. [3] Signal shadowing due to obstacles is too high to be compensated by forward error correction (FEC) thus leaving its focus on the attenuation caused by rain.

As rain attenuation appears only from time to time and its maximum fade slope value for $0.01 \%$ of an average year is about $0.6 \mathrm{~dB} / \mathrm{s}$, adaptive forward error correction and also adaptive modulation techniques are applied to efficiently use the available bandwidth. This technique is a major improvement in contrast to traditional systems which required a rather high link margin which unnecessarily delimits up- and downlink capacity. The employed FEC scheme guarantees a fixed useful data rate by adapting the MAC packet lengths according to the used coding and modulation scheme. ${ }^{1}$ Figure 3 illustrates the resulting up-link availability for which the cell error rate (CER) does not exceed the threshold of $10^{-6}$ and shows that for $99.14 \%$ of the time, no FEC is necessary and that an availability of $99.92 \%$ is achieved by adaptive FEC with a $\mathrm{RS}(65,53)$ code and a rate $1 / 2$ block turbo code (at an elevation of $\left.20^{\circ}\right){ }^{2}$ Additionally, the adaptive FEC scheme accounts for the influence of the drastic change of the elevation angle which in turn influences the effects of rain. For rainless periods and a high elevation angle, as depicted in Fig. 4, a 16 QAM modulation without FEC is sufficient for an ATM CER of less than $10^{-6}$ whereas only for heavy rain intensities and low elevation angles QPSK with RS and Turbo Code has to be turned on.

\section{PRotocol Implementation And TESTS}

The designed protocol is implemented using the FreeBSD5.0-current version [4] for testing and demonstration purposes. The implementation is modular in terms of separating a satellite channel emulation entity from the implementation of the MAC protocol. Therefore it is possible to run tests over various satellite constellations ${ }^{3}$ besides the original LEO target system.

\footnotetext{
${ }^{1}$ The design supports also a fixed MAC packet length by adapting the useful data rate even though this option is not implemented in the demonstrator.

${ }^{2}$ The reference constellation of the system is here defined as 72 satellites in 12 orbits with an orbit height of $1350 \mathrm{~km}$ and an inclination of $47^{\circ}$.

${ }^{3}$ In fact, the same environment is currently used to evaluate connecting the ISS' Columbus module via ATM to Earth.
} 


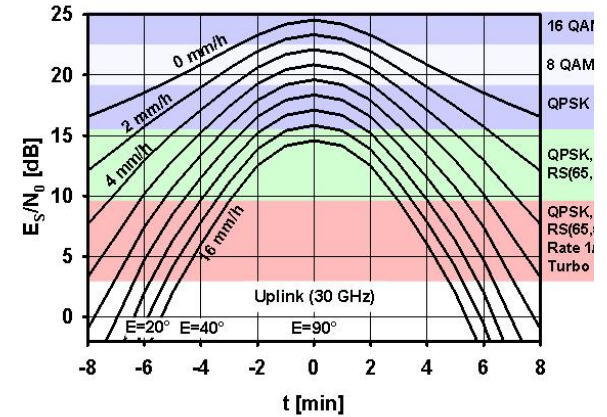

Fig. 4. TDMA Scheduler \& MAC Up- and Down-link Frame Structure

\section{A. Demonstration Environment}

The demonstrator is separated into three major components (Fig. 5): a satellite channel emulator (SCE), satellite and ground terminal DLCs (SDLC \& TDLC), and a control station (CS). The ATM switch is only used for measurement purposes on ATM link level; the terminal can be any ATM-equipped computer.

1) Satellite channel emulator: The SCE is configurable via snmp and adds the variable propagation delay and error rates depending on the current elevation angle. STK-based AER and error probability tables are used as an input file. Additionally it implements contention for terminals writing into the same MAC time slot. Its functionality is independent of the network interface used to connect DLC and SCE. Due to implementation purposes, Ethernet is used for encapsulating the actual MAC frame structure.

2) Satellite and Ground Terminal DLC: The SDLC and TDLC units implement the MAC functionality. They are separated from the SCE in order to be independent of satellite specific network characteristics.

3) Control Station: The CS initializes the SCE and DLC entities and acts additionally as a NTP-Server which is used by the latter two units for time synchronization.

\section{B. Measurements}

After an extensive simulation phase of the MAC [5], [6], FEC schemes [7], and upper layer protocols [8], [9] over the proposed system architecture, measurements to verify the functionality are conducted. The SCE is well capable to merge the different up-link streams coming from each TDLC into a single up-link stream going to the SDLC (including the contention handling) in real-time. The added variable propagation delay is shown in Fig. 6. The mean packet delay follows exactly the expected theoretical value; the pikes of
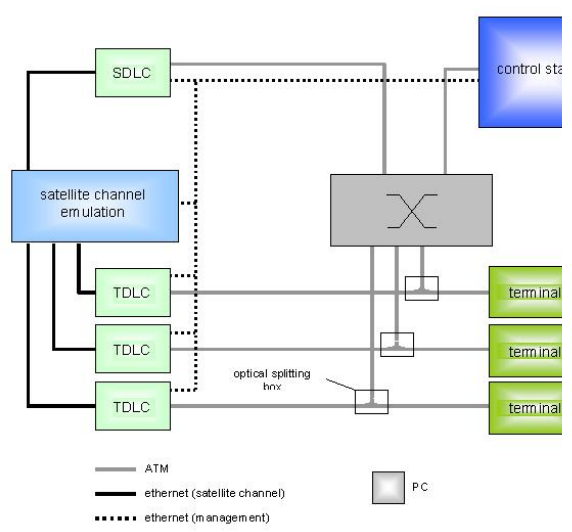

Fig. 5. Demonstrator Environment Overview

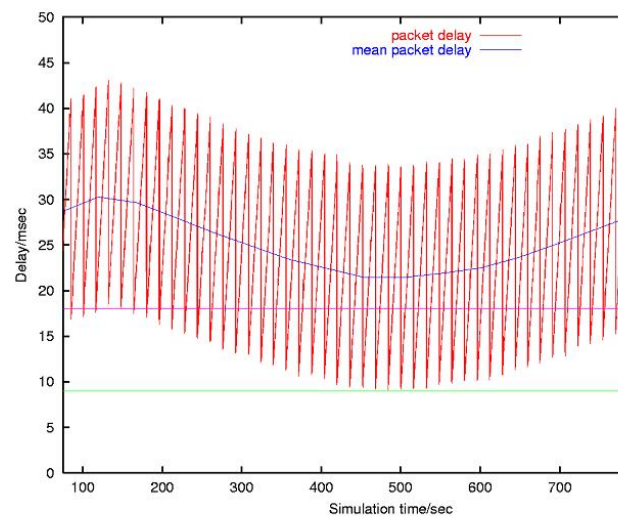

Fig. 6. Measured One Cell Delay

the current cell delay are caused by the $24 \mathrm{~ms}$ framing (a cell may either be directly transmitted or has to wait for the next up-link frame) and the constant shift is due to processing delays. Figure 7 shows the error rates for various coding schemes (CRC, RS, and Turbo) and rain intensities (6, 10, and $16 \mathrm{~mm} / \mathrm{h})$. The measurement shows clearly the advantage of switching the FEC scheme on the fly during the connection.

\section{Conclusion}

The presented QoS aware MAC protocol suitable for ATM based NGSO satellite networks is adaptable to the changing satellite channel characteristics in terms of elevation angle dependent attenuation and the resulting BER. It allows optimal 


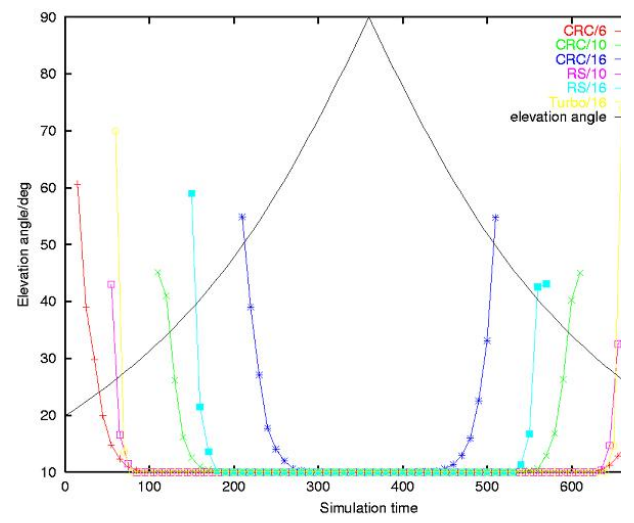

Fig. 7. Measured Error Rates for Various Codings and Rain Intensities

link utilization by changing its FEC scheme on the fly; dynamic MAC packet lengths guarantee a fixed useful data rate. The partial protocol implementation and measurements show the advantage of its adaptiveness.

\section{REFERENCES}

[1] H. Bischl, H. Brandt, A. Dreher, M. Emmelmann, F. Krepel, E. Lutz, L. Richard, P. Todorova, and M. Werner, "ATM-based multimedia communication via NGSO-satellites," unpublished.

[2] H. Bischl, H. Brandt, A. Dreher, M. Emmelmann, A. Freier, B. Hespeler, F. Krepel, E. Lutz, W. Milcz, L. Richard, P. Todorova, and M. Werner, "ATM-sat: ATM-based multimedia communication via LEO-satellites," German Aerospace Agency (DLR)," Final Report, Dec. 2002.

[3] C. Hunscher, R. Mayer, A. Jacob, L. Stange, A. Dreher, and L. Richard, "Aktive antenne für multimediakommmunikation über satellit," Technische Universität Braunschweig, Germany, Final Project Report BMBF contract no. 50 YB 0004, 2001.

[4] (2003) The freebsd homepage. [Online]. Available: http://www.freebsd.org/

[5] J. Bostic, "Report on OPNET simulatoins," German Aerospace Agency (DLR), Oberpfaffenhofen, Germany, Tech. Rep. DLR-IB, IB 554-01/02, 2002.

[6] - "Broadband wireless access MAC simulator," in Proc. of the 9th Electronical and Computer Conference ERK 2000, Portoroz, Slovenia, Sept. 21-23, 2000, pp. A-147 - A-150.

[7] H. Bischl, "Availibility and error control for ATM via satellite at KaBand," presented at the Deutscher Luft- und Raumfahrtkongress 2001, Hamburg, Germany, Sept. 2001.

[8] M. Emmelmann. (2002, May) Dimensioning of receive buffer size and timer granularity for optimal network performance of TCP in a variable delay leo satellite network. NASA Space Internet Workshop '02. [Online]. Available: http://siw.gsfc.nasa.gov

[9] - "Effects of advertised receive buffer size and timer granularity on TCP performance over erroneous links in LEO satellite networks," in Proc. IEEE Globecom '02, Taipei, Taiwan, Nov. 17-21, 2002. 